\title{
US FDA/EMA harmonization of their bioanalytical guidance/guideline and activities of the Global Bioanalytical Consortium
}

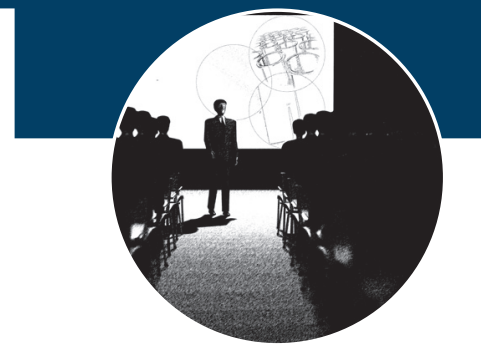

\section{I American Association of Pharmaceutical Scientists Annual Meeting Washington DC, USA, 26 October 2011}

The 2011 annual conference of the American Association of Pharmaceutical Scientists, held in Washington DC, USA, hosted a roundtable entitled: 'Update of the US FDA/European Medicines Agency (EMA) harmonization of their bioanalytical guidance - Global Bioanalytical Consortium activity and impact on small and large molecules.' The roundtable was initiated with a presentation from CT Viswanathan on the history of the revision of the FDA guideline on bioanalytical method validation. It was followed by a presentation by Jan Welink who presented an update on the final European Medicines Agency guideline on bioanalytical method validation with relevance to ongoing harmonization efforts. The final presentation was by Fabio Garofolo on the progress of the Global Bioanalytical Consortium harmonization teams for small and large molecules. Brian Booth and Sam Haidar of the FDA updated the audience on the status of the revision of the FDA bioanalytical guidance. The roundtable was moderated by Stephen Lowes.

This roundtable was conducted as part of the 2011 American Association of Pharmaceutical Scientists (AAPS) conference in Washington DC, USA. The session was a unique opportunity for participants involved in generating preclinical and clinical bioanalytical data for bioavailability (BA), bioequivalence (BE) and pharmacokinetics (PK) to have an update on the current status of the US FDA guidance and European Medicines Agency (EMA) guideline on bioanalytical method validation (BMV), and also on the progress of the Global Bioanalytical Consortium (GBC).

Approximately 400 global professionals from the bioanalytical field attended.

\section{History of the FDA guideline on BMV \& harmonization \\ CT Viswanathan (CT Viswanathan and Associates, Inc., USA) opened the roundtable by a lecture on harmonization and globaliza- tion of the bioanalytical guidance. He started his presentation by mentioning that the views expressed are his own and do not reflect offi- cial policy of the FDA. The presentation con- tinued with a review of the last 20 years of leadership and history of the FDA in the bio- analytical industry. During his presentation, Viswanathan mentioned that the past AAPS/ FDA Crystal City workshops [1,2] have estab- lished the basic principles of industry practice in bioanalysis. Over this time period, the FDA}

has gained experience and tried to understand industry practices, sharing information and contributing to open discussions with scientists in the industry. Moreover, he explained that he developed a compliance program for BE inspections at the FDA. Now, the agency has many years of experience in compliance inspections, in training their inspectors, and has built an exhaustive database of inspections performed all over the world. All of these inspections provided data and insights into how their bioanalytical guidance could be improved and how to cover new areas in the bioanalytical field.

The FDA started the review process of their existing BMV guidance [3] used for bioanalytical work, and Viswanathan stated that this revision process is ongoing. He reported that the current trend in the pharmaceutical industry is to outsource more bioanalytical work and more studies are submitted to multiple regulatory agencies all over the world. This trend leads to discussions on the harmonization of the present guidance to overcome difficulties in complying with many different regulatory guidelines. Presently, the BMV guidance from the FDA [3] is the most recognized document throughout the bioanalytical industry. Although this guidance, issued in 2001, needs to be updated to reflect the evolution of the field through the years, it has already brought some uniformity to the bioanalytical community. Recognizing
Fabio Garofolo*1, Josée Michon', Virginie Leclaire', Brian Booth', Stephen Lowes ${ }^{3}$,

CT Viswanathan ${ }^{4}$, Jan Welink ${ }^{5}$, Sam Haidar ${ }^{2}$, Leonardo de Souza Teixeira ${ }^{6}$, Daniel Tang ${ }^{7}$, Binodh Desilva ${ }^{8}$

'Algorithme Pharma Inc., Laval (Montreal), QC, Canada ${ }^{2} U S$ FDA, CDER, Silver Spring, MD, USA

${ }^{3}$ Advion BioServices, Ithaca, NY, USA

${ }^{4} \mathrm{CT}$ Viswanathan \& Associates Inc., USA

${ }^{5}$ Medicines Evaluation Board, The Hague, The Netherlands ${ }^{\prime} \mathrm{CF}$, Goiania, Brazil

${ }^{7}$ ICON APAC, Shanghai, China ${ }^{8}$ Bristol-Myers Squibb, Princeton, NJ, USA

*Author for correspondence:

Tel.: + I 4509736077

Fax: + | 4509732446

E-mail: fgarofolo@algopharm.com 
that, in July 2011 the EMA issued its own BMV guideline [4] and the interactions between the FDA and EMA will be very crucial to align their two respective guidelines before the issuance of the updated FDA guidance. Viswanathan mentioned that the core of both FDA and EMA guidance documents are very similar, therefore the harmonization process is expected to be smooth.

Viswanathan stated that the globalization of the guidance is different from its harmonization. Globalization will require acceptance from everybody to form a unified single bioanalytical guidance, which will make this process significantly complex. He referenced his proposition made at the $4^{\text {th }}$ Workshop on Recent Issues in Bioanalysis (4th WRIB) in Montreal in 2010 [5] and reiterated at the $1^{\text {st }}$ Asia Pacific Bioanalytical Conference (1st APBC) [6] in Shanghai in 2011 to form a global bioanalytical consortium. Acknowledging that this is now taking shape, Viswanathan concluded that this consortium could be very useful for the regulatory authorities as a potential tool for future globalization.

\section{The final EMA guideline on BMV \& harmonization}

Jan Welink (EMA/Dutch Medicine Evaluation Board, The Netherlands) started the presentation with a disclaimer that the views expressed were his own and do not reflect official policy of the EMA.

Two major topics were discussed regarding the final EMA guideline on BMV [4]: how it was harmonized between European countries, and how it was intended to be part of global harmonization.

There was a need for an EMA guideline on $\mathrm{BMV}$ as no pan-European guideline existed to regulate bioanalytical work submitted to European agencies. Therefore, efforts have been made to provide a EMA guideline for BMV. The major group involved in directing this process was the Pharmacokinetics Working Party (PKWP).

Welink stepped the audience through the EU guideline development process to facilitate harmonization within Europe. In December 2008, the concept paper was written and submitted for public consultation until March 2009 A draft guideline was then publicly issued in December 2009, followed by a public comment period through May 2010. The final guideline was then written and issued in July 2011, effective 1 February 2012, along with the overview of received comments. Once again, the group coordinating the process was the PKWP.

The EMA guideline on BMV was drafted by representatives from France and The Netherlands. Discussion and agreement on the draft guidance was then made by PKWP members from nine European states.

Scientific discussions on the EMA guideline on BMV were conducted in Europe in the presence of formal representation of EMA/ Committee for Human Medicinal Products (CHMP) at the The European Bioanalysis Forum (EBF) and European Federation for Pharmaceutical Sciences (EUFEPS) conference in Brussels (April 2010). The new EMA guidance was accepted by all European member states. Welink clarified that the new EMA guidance was also created with global harmonization intent. In fact, the second public consultation in May 2010 on the draft guideline resulted in comments received from over 50 industry and regulatory sources (variety of stakeholders including industry and other regulatory bodies) from the Americas (North, Central and South), Africa (Middle-East) and Asia, in addition to those received from Europe. These comments were discussed and taken into account where considered applicable and/or necessary. As part of the process, there were direct communications with the FDA, both informal and formal. These discussions between the EMA and FDA took place under confidentiality agreements.

Also, scientific discussions on the EMA guideline on BMV were held in formal representation of EMA/CHMP in numerous international conferences outside Europe: 4th WRIB, April 2010, Montreal, Canada; National Biotechnology Conference (NBC) meeting, May 2010, San Francisco, USA; AAPS meeting, November 2010, New Orleans, USA; 1st APBC January 2011, Shanghai, China [6].

The EMA guideline on BMV took into account the FDA BMV guidance for industry (2001) [3], different published papers, such as the AAPS/FDA Crystal City III White Paper [1] and AAPS workshop on incurred sample reproducibility [2], and other recent White Papers, as well as EMA experience based on issues seen during review of dossiers and inspections.

Finally, Welink discussed the next steps following the publication of the final EMA guideline. He stated that the EMA is supportive of the GBC objectives and anticipate productive interactions with the consortium. He reminded delegates that the GBC should not only focus on FDA/EMA regulations, but 
should also take into consideration a worldwide agreement. Furthermore, the next steps after the GBC output will be of crucial importance: what to do with the outcome/output of GBC, and how to get the regulatory agencies involved. He concluded by asking "Who will take the lead once the GBC recommendations have been received?"

\section{Update on the progress of the GBC harmonization teams (GBC-HT) for small \& large molecules}

The third presentation of the meeting was from Fabio Garofolo (Algorithme Pharma Inc., Canada) and was entitled: 'Update on the progress of the Global Bioanalytical Consortium harmonization teams (GBC-HT) for small and large molecules.'

This presentation began with a brief history of the GBC [7]. The first discussions about guideline harmonization were started in 2008-2009. The discussion became more serious at the $2009 \mathrm{EBF}$ conference in Barcelona where discussion among international scientific organizations took place: the AAPS, Applied Pharmaceutical Analysis (APA), Calibration and Validation Group (CVG), and the EBF. The initiative was continued with a letter sent to the FDA and EMA in February 2010 and the publication of an open letter in the April 2010 edition of Bioanalysis [8]. The creation of the GBC was announced during the 4th WRIB held in Montreal (Canada) [5].

Garofolo explained the organizational structure of the GBC. A GBC steering committee (GBC-SC) was formed to support a Scientific Leadership Team (GBC-SLT), which in turn guides multiple harmonization teams. The steering committee is composed of ten members from various regions of the world: North America (3), Latin America (1), Asia (3) and Europe (3). These members provide organizational support and facilitate communication pathways to the different harmonization teams. The teams are divided into three different areas: 'Topics common to all molecules,' 'Large molecules/ligand binding assays' and 'Small molecules/chromatographic assays.'

Through its deliberations, the GBC-SC developed new insights for the upcoming GBC work. The GBC-SC is now working on new initiatives to support the harmonization process and will promote increased engagement, input and contribution from different regions of the world. Focus is to provide opportunities for regular and detailed updates on the GBC progress to the global bioanalytical community as a whole. Garofolo concluded that the GBC global meeting is planned the week of 24 September 2012 in Europe.

\section{Roundtable}

The three presentations were followed by a roundtable, where comments and questions from the attendees were welcomed. The participant questions were addressed by a panel composed of Brian Booth and Sam H Haidar (FDA, USA), CT Viswanathan, Jan Welink, and Fabio Garofolo. The roundtable was moderated by Stephen Lowes (Advion BioServices, USA).

Q Is there any plan for comments from the community on the draft FDA guidance?

Booth responded that, indeed, it will be available on the FDA website for comments for a period of 90 days.

\section{How can Latin America GBC}

representative ensure that all Latin America countries are aware \& contribute to the GBC initiative?

Leonardo de Souza Teixeira (ICF, Brazil), answered that there will be an important conference in November for South America (MERCOSUL).

Q How do the FDA \& EMA view the efforts \& objectives of the GBC?

Welink emphasized the fact that the EMA supports the harmonization process and the GBC objectives. The approach should incorporate scientific thinking, as well as regulatory thinking. He clarified that the final decision will be taken by regulators, but a successful GBC outcome will be very influential in the process. Haidar continued by saying that the GBC work will be valuable in identifying issues and resolutions and anticipated the new FDA guidance will reflect such input from the scientific community. Booth also commented that the role of the GBC is important, because the industry must be unified on the particular issues of validation in order for regulatory groups to address them. Otherwise, there will be disparate views from different areas that will be difficult to reconcile via harmonization. This initiative could be helpful in the long run. Finally, on this question, Viswanathan responded that the GBC is taking on a huge task, but as long as everyone remains open to the different perspectives, the process is very valuable to the harmonization objective. 
Q Is there any collective feedback on how regional authorities other than the FDA \& EMA view the efforts and objectives of the GBC?

Garofolo, in agreement with de Souza Teixeira, answered that Brazil ANVISA strongly supports the GBC initiative. Garofolo continued that Japan authorities are supporting the newly formed Japan Bioanalysis Forum (JBF) and its link to GBC. There is no feedback from India and China at this point and this needs to be pursued.

The GBC harmonization team work was only started within the past 8 months, \& is planning to have completed its recommendations in late 2012 after the global meeting in September. How can it be done with such a quick turnaround? Garofolo pointed out that the different teams have established good rapport with each other and this is facilitating effective decision making. It was acknowledged that some groups are progressing faster than others but timeline milestones are being set by the GBC-SC with support to help efficient progress. There are emerging opportunities for the community to participate and comment and it is expected that this will help the teams further optimize their efforts.

There are many opportunities for the GBC to get comments from the scientific community, but it seems like the regulatory agencies are not really involved. Why not? There are so many people, so many different countries with so many opinions, which organization will choose \& decide the final document for harmonization?

Viswanathan responded that the current focus of the regulatory agencies, FDA and EMA is to harmonize the existing guidances between them and they have not looked beyond. Future efforts must deal with globalization, which no doubt will be challenging. It is imperative that the GBC must work together actively with all regulatory agencies in having them adopt an unified single guidance. Garofolo added that the GBC is a scientific group. The guidance will have to be reworded by the regulators.

Q Are there a lot of bioanalytical activities in China?

Garofolo mentioned that it seems there is a good participation from China, demonstrated by the active and wide participation of the Chinese bioanalytical community, and China SFDA support to the 1st APBC in January 2011 in Shanghai. Daniel Tang (ICON APAC) added that in China the new bioanalytical guidance is being written by technical people and then adopted by the agency. The draft guidance of the new Chinese Pharmacopeia (2015 version) has already included updates from the new EMA BMV guideline.

\section{Who is financing the GBC?}

Garofolo simply answered: "Nobody right now!" Major pharmaceutical companies are taking care of all the teleconferences cost. Everyone is giving their own time and efforts on voluntary basis since each harmonization team manager has its own milestones to achieve. However, funding is welcome, especially for traveling expenses.

Q Assuming there will be differences between the EMA guideline and the upcoming publication of the revised FDA guidance, what would be a projected path forward to resolve, integrate \& unify?

Welink answered that EMA and FDA should discuss, but there are no huge differences expected. If something was missed in the EMA guidance, a clarification could be issued. However, there may be some minor differences between the FDA and EMA guidelines since the FDA and EMA opinions differ on some topics. The EMA represents 27 member states in Europe and is allowed to make its own recommendations. Haidar responded that there should be only minor differences between the two guidance documents. Since the FDA guidance is not yet finalized, clarifications and discussions could still be done if needed. Booth agreed that there will be no major differences. Welink added that there are already some areas that are known to be slightly different. All the regulators in the panel emphasized that the guidance is intended to be clear on what to do or not, but they are still recommendations with latitude to adjust according to scientifically defendable needs.

Q After the September 2012 global meeting, what are the next steps once the GBC recommendations have been completed? Garofolo said that a White Paper is to be published and the GBC hopes that the recommendations will be taken into consideration by the regulatory agencies. Mark Arnold (Bristol-Myers Squibb, Princeton, NJ, USA) added that regulatory agencies should reach out to the GBC during the process and GBC representatives should meet agencies from more countries. Everyone should start working together. 
Can a central regulatory mechanism/body be created to attend to the harmonization of bioanalytical guidance between the FDA \& EMA?

Welink answered that there is already discussions between the FDA and EMA on certain topics. There are certainly efforts made on coordination. Haidar added that limited resources at the FDA is a major challenge.

Q How is the GBC planning to integrate its role in the FDA/EMA harmonization process?

Garofolo said that the GBC mission is to issue its scientific-based recommendation as soon as possible to try to influence the agencies since some regulatory agencies besides the FDA and EMA are already drafting their bioanalytical guidance (Brazil-ANVISA, China-SFDA, Japan-MHLW, Canada-TPD). Regulatory agencies are welcome to interact with the GBC now as the timelines are really short.

2 Assuming there is some natural 'turnover' in the GBC-HT teams, is there any opportunity for those coming into the process after the start to volunteer?

Garofolo responded that the momentum is already ongoing and new members may slow down the teams. However, the whole community is welcome to be involved in regional meetings or on the upcoming interactive GBC website.

Q In light of the GBC process, how will the regulatory agencies continue to adapt and evolve their guidance documents?

Welink mentioned that the GBC process should be ongoing since there are always new guidelines and new techniques. Mark Arnold added that if people participate in AAPS meetings and other conferences, they will be heard and their representative will bring their ideas to the other GBC members.

Q Are there differences between the FDA \& EMA guidelines that are not negotiable? Booth said that the FDA commented on the EMA guideline when it was drafted. However, there will always be potential differences between the guidelines.

What about biomarkers?

Booth answered that there will be differences between biomarkers validation guideline and what is currently done for validation of small molecules methods. However, the guideline will only be descriptive. Viswanathan added that the success will be dependent on the cohesion between the FDA and EMA. They will discuss but it will take time. Issues will come from other countries and regulatory agencies. We should invite them to build up a relationship now. But, unfortunately, it will not be possible to get in touch with all countries.

\section{Conclusion}

In summary, this roundtable on the update on the FDA/EMA harmonization of their bioanalytical guidance/guideline and on GBC activities was very interactive and brought together an exceptional panel for the roundtable session format. The great success of this roundtable confirms the keen interest of the industry and the regulatory bodies worldwide to work together for the harmonization and globalization of bioanalytical guidances.

\section{Acknowledgements}

A special thank you to: Mark Arnold (Bristol-Myers Squibb) for the critical review of this document, stimulating discussions and knowledgeable comments; Josée Michon and Virginie Leclaire (Algorithme Pharma Inc.) for writing the first draft of this document and taking the minutes of this roundtable; Suzanne Martinez, Isabelle Dumont (Algorithme Pharma Inc.) and Wei Garofolo (Calibration and Validation Group) for the revision and correction of this document; Bethany Daniel (American Association of Pharmaceutical Scientists) for her great help in organizing this successful roundtable session during the 2011 American Association of Pharmaceutical Scientists Annual Meeting.

\section{Disclaimer}

The views expressed in this article are those of the authors and do not reflect official policy of the US FDA, Medicines Evaluation Board and European Medicines Agency. No official endorsement by the FDA, Medicines Evaluation Board and European Medicines Agency is intended or should be inferred.

\section{Financial \& competing interests disclosure}

The authors have no relevant affliations or financial involvement with any organization or entity with a financial interest in or financial conflict with the subject matter or materials discussed in the manuscript. This includes employment, consultancies, honoraria, stock ownership or options, expert testimony, grants or patents received or pending, or royalties.

No writing assistance was utilized in the production of this manuscript. 
